\title{
SOFTWARE AS A SERVICE FOR EFFICIENT CLOUD COMPUTING
}

\author{
K.V. K Mahesh Kumar \\ Research Scholar, Department of Computer Science and Engineering,Acharya Nagarjuna University, A.P, India
}

\begin{abstract}
This Research paper explores importance of Software As A Service (SaaS) for efficient cloud computing in organizations and its implications. Enterprises now a days are betting big on SaaS and integrating this service delivery model of cloud computing architecture in their IT services. SaaS applications are service centric cloud computing delivery model used as IT Infrastructure which is multi-tenant architecture used to provide rich user experience with desired set of features requested by the cloud user. This research paper also discusses the importance of SaaS application architecture, functionality, efficiency, advantages and disadvantages.
\end{abstract}

Keywords: Cloud Computing, Service Delivery Models, Software as a Service, SaaS Architecture. $* * *$

\section{INTRODUCTION}

The Word Cloud Computing is buzzing everywhere among organization, enterprises, independent software vendors (ISV), end users etc. Cloud computing is nothing but distributed computing over the internet where user can access their data from the database in the cloud. Cloud computing is different from traditional grid computing it is more dynamic, flexible and scalable offered by independent organizations where deployment and maintenance of the services \& data is managed by the organizations themselves. Cloud computing varies from one cloud provider to another, as some cloud providers provide storage over network with small monthly rentals for end users, whereas some other providers offer applications for software companies which helps in reducing costs in deployment or installations of applications. In this paper we will be evaluating SaaS service delivery model, its architedctural impact, characteristic features and providing solutions for businesses with the integration Application program interface (API) [1], [2], [9].

\section{OVERVIEW OF CLOUD COMPUTING}

Cloud computing is known for applications delivered as services over the Internet and the hardware and systems software in the datacenters that provide those services. There are four basic cloud delivery models

\subsection{Private Cloud}

In this cloud services are provided solely for an organization and are managed by the organization or a thirdparty. These services may exist off-site [1], [8].

\subsection{Public Cloud}

Public cloud services are available to the public and owned by an organization selling the cloud services. For example storage over networks such as Drop box, Google drive etc [1], [8].

\subsection{Community Cloud}

In Community cloud services are shared by several organizations for supporting a specific community that has shared concerns such as common goal, security requirements, policies, compliance considerations etc. And these services are further managed by a third party organization which may exist at an offshore location [1], [8].

\subsection{Hybrid Cloud}

Hybrid cloud is a composition of various cloud computing infrastructures such as public, private or community. For example hybrid cloud is the data stored in private cloud is manipulated by a program running in the public cloud [1], [8].

The cloud computing architecture is further classified based on service delivery models, well there are three service delivery models namely Software As A Service (SaaS), Platform As A Service (PaaS) and Infrastructure As A Service (IaaS) [1], [8].

\section{SOFTWARE-AS-A-SERVICE (SaaS)}

Generally cloud providers use public cloud resources to create their virtual private cloud to make of cloud computing access the scalable computing resources and IT services. SaaS is one of the service delivery models where of software as a service will change the way people build, sell, buy and use software. In this model Software is provided as a service where cloud user can access the software from his web browser without the 
concerns of deployment or installation \& maintenance. SaaS applications are known as Web-based software, on-demand software or hosted software. Cloud provider maintains the application its security, availability and performance. SaaS cloud computing delivers end user desired application through the internet to thousands of customers using a multitenant architecture. On the other side cloud user can run application or software with no upfront costs or investment in database, servers and software licensing. With the advent of Web 2.0 \& faster HTML 5 standards, graphically rich applications can be run smoothly at 60 Frames per second just like running our software on our own personal computers. This service model depicts one to many function as single application running as a service on the server side with many client end users can run it from their web browser simultaneously as they are connected as services [2], [4].

For example let us consider Google Play Store where applications can be purchased on the go and can be run from the web browser, any number of people can purchase and run the application simultaneously but only one instance of the application is running on the server side. This helps in reducing costs on resources and on the customers point of view it is hassle free process service on demand where Cloud user can rent applications without the need of installation and maintenance of the software [2], [4].

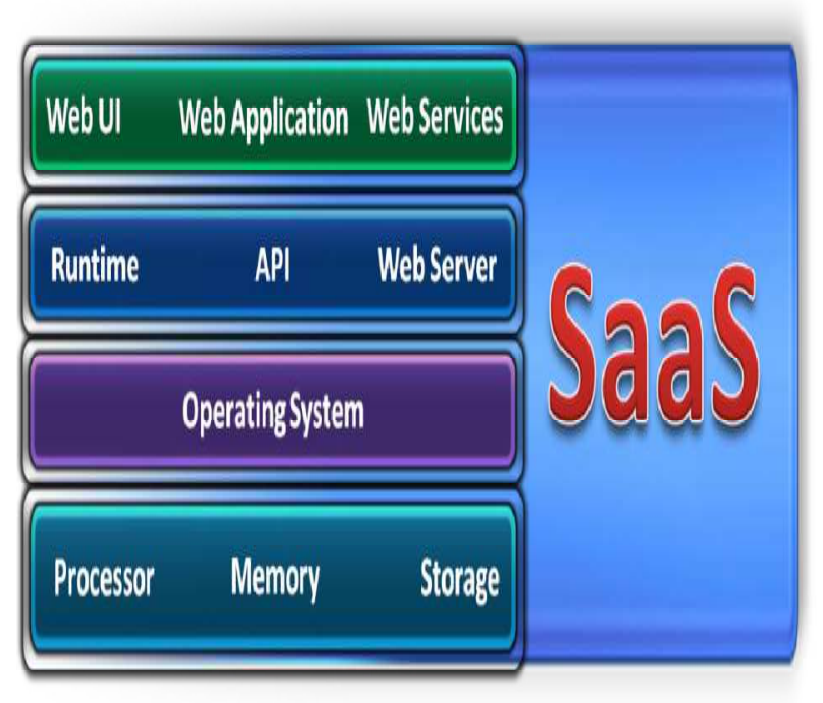

Fig-1:SaaS Structure [6]

\subsection{SaaS Architecture}

Software as a Service (SaaS) has a distinctive advantage of Service Oriented Architecture where software applications communicate with each other. An application running as a service act as a service provider and exhibits its functionality to other applications or services via public brokers and also acts as a service requester when required for incorporating data and functionality from other services. SaaS service delivery model system architecture supports user demands even at peak hours and has the ability to process large numbers of transactions in a secure and reliable environment[7], [10].

\section{software-as-a-service model}

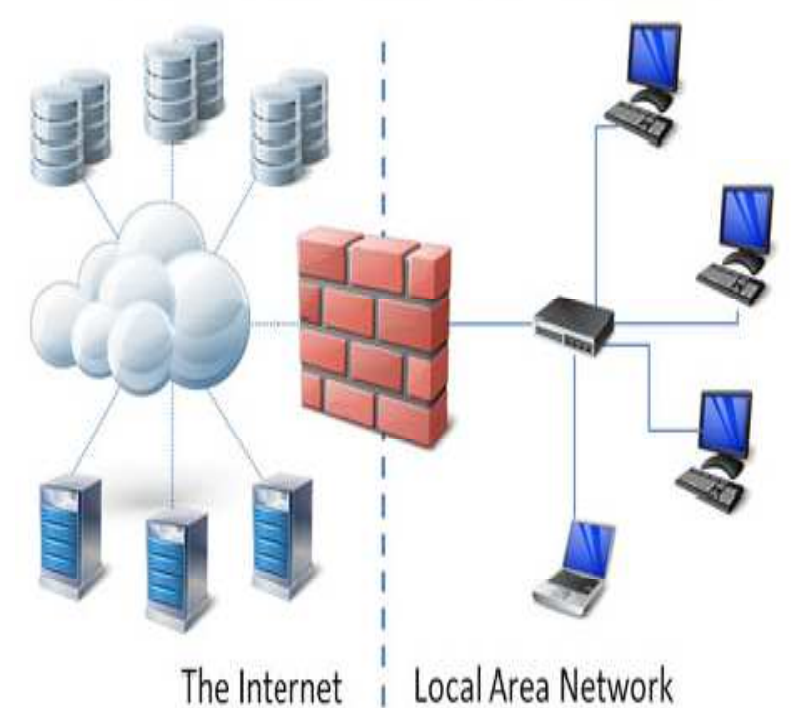

Fig-2:SaaS Architecture [6]

SaaS with the use of new technologies and application frameworks helps in reducing time to market and cost savings in converting on premises server into a SaaS based product. Microsoft believes SaaS architecture can be classified based on their maturity levels and they are as follows:

\subsubsection{Ad-hoc/Custom}

Ad-hoc or custom level is the first level of maturity where unique or customized version of applications are hosted on the servers. This level is useful when migrating from or converting existing client server architecture. As it doesn't requires system administrator which indeed helps in reducing maintenance costs [7], [10].

\subsubsection{Configurability}

This second level of maturity helps in providing flexibility in identifying different users using the same application or service. This is done by configuring unique metadata, which indeed helps cloud provider in identifying different user and their needs, So that cloud provider can maintain common core code of the application regardless of end users and their needs. Further it also helps cloud provider in allocating the resources 
such as application or software based on end user demands [7], [10].

\subsubsection{Multi-Tenant Efficient}

Multi-tenancy is known for sharing of resources across hundreds of tenants or end users but can still differentiate individual users, their data and needs or demands [7], [10].

\subsubsection{Scalability}

In this level of maturity application resources are used efficiently by conducting best practices of IT such as optimizing locking duration, statelessness, sharing pooled resources such as threads and network connections, caching reference data and partitioning large databases [7], [10].

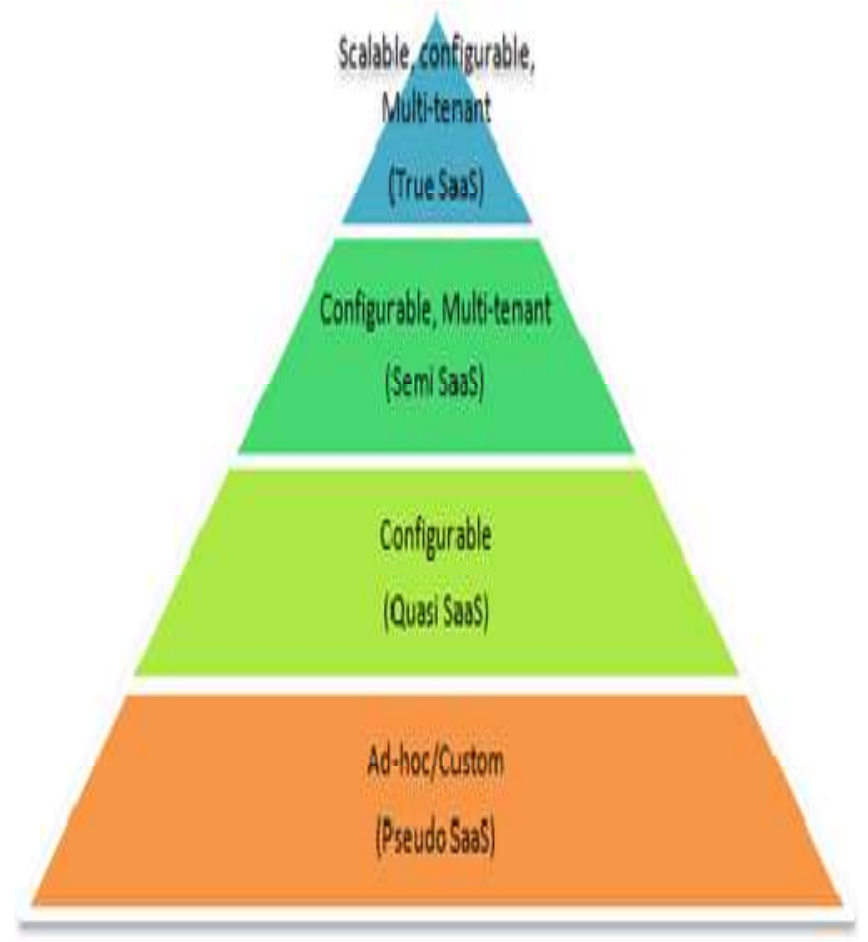

Fig-3:SaaS Various Types [6]

\subsection{Characteristic Features of SaaS}

Software as a Service has three prominent Characteristic features they are

\subsubsection{Network or Online Access}

SaaS is an online application which can be accessed using a web browser from anywhere in the world without any software installation. All we need is internet access and a web browser with which the cloud provider services and applications can be accessed [3]

\subsubsection{Centralized Management}

SaaS service model major characteristic feature is centralized management, which will help monitoring, controlling, maintaining and updating applications at appropriate time without any hiccups. The maintenance of the application is done at the offshore location or at the cloud provider premise where end user or cloud user does not need to worry about updating the application [3].

\subsubsection{Powerful Communication Features}

SaaS not only known for its online services \& application but also has powerful communication features. SaaS is known for its Instant Messaging chat service and Voice over IP (VOIP) features [3].

\subsection{Advantages of SaaS}

Advantages of Software as a service (SaaS) are classified into two categories Cloud user or end user advantages and Cloud provider advantages.

\subsubsection{Cloud User Advantages}

- Offsite deployment

- Low overhead or low costs

- Decentralized

- Customizable

- On the fly pay as u go

\subsubsection{Cloud Provider Advantages}

- Application as a service

- Scalable applications

- High customization

- Highly stable \& common base code

- Easy maintenance

- Maximum efficiency

- Flexible costs based on usage

\subsection{Disadvantages of SaaS}

\subsubsection{Strong \& Reliable Network Connectivity}

SaaS is a service model where the cloud user can access the application through web browser, this means we need a good reliable \& fast internet connection. But internet speeds and connectivity differ from place to place, country to country. So strong \& reliable network connectivity can be a big disadvantage [6].

\subsubsection{Security Issues}

SaaS has increased security issues and challenges as it constantly interacts with different cloud users or end users, data security and integrity is at stake. As one service is shared by many cloud users [6]. 


\subsubsection{Load Balancing Feature}

Load balancing is one of the challenges in cloud computing which can be mitigated by businesses or cloud providers by consistent monitoring of all SaaS applications. Currently only major player like Apple, Microsoft, Google etc. are offering load balancing [6].

\section{EFFICIENT SAAS WITH API INTEGRATION}

Software-as-a-service (SaaS) is a contemporary cloud computing trend emerging in the IT industry which is getting better day by day and a preferred choice of service delivery model for cloud providers. Biggest advantage of SaaS cloud computing service delivery model is the more meaningful, powerful and efficient integration of Application Programming Interface (API). Now a days Business needs or demands are evolving day by day, in order to meet these demands from their customers or end users, cloud providers need to maintain \& utilize their resources efficiently for providing services they are confined with. In order to meet these challenges businesses needs to update their applications $\&$ services with the pace of the new technology. Coding applications with new technologies available from time to time is a costly assignment for cloud providers. This is where API plays a major role in mitigation of additional costs occurring in developing new apps. Instead of rewriting the app completely businesses will just add new API's in their existing application. API integration is easy and efficient for adding additional features from new technologies into their existing services and applications [5].

\section{CONCLUSION}

Enterprises would do well to consider the flexibility and riskmanagement implications of adding SaaS to their portfolios of IT services. Integration and composition are critical components in your architecture strategies to incorporate SaaS successfully as a fully participating member of your servicecentric IT infrastructure. Today, SaaS applications are expected to take advantage of the benefits of centralization through a single-instance, multi-tenant architecture, and to provide a feature-rich experience competitive with comparable on-premise applications. A typical SaaS application is offered either directly by the vendor or by an intermediary party called an aggregator, which bundles SaaS offerings from different vendors and offers them as part of a unified application platform.

\section{REFERENCES}

[1]. Armbrust, M et.al 2010, "A View of Cloud Computing", Communications Of The ACM, Vol. 53, No.4, pp. 50-58.
[2]. Bento, Al, 2011, "Cloud Computing: A New Phase in Information Technology Management", Journal Of Information Technology Management, Vol. 22, No. 1, pp. 39-46.

[3]. Chou, W 2008, "Web Services: Software-as-a-Service (SaaS) Communicaton and Beyond", IEEE Congress on Services.

[4]. Godse, M \&Mulik, S 2009, “An Approach for Selecting Software-as-a-Service (SaaS)”, IEEE International Conference, pp.155-158.

[5]. Javier, E, David, C \&Arturo, M 2008, “Application Development over Software-as-a-Service Platforms". IEEE international conference, pp.97-104.

[6]. Kulkarni, G, Gambhir, J \&Palwe, R 2012, "Cloud Computing-Software As Service”, International Journal of Cloud Computing And Services Science, Vol. 1, No. 1, pp.11-16.

[7]. LIAO, H 2009, "Design of SaaS-based Software Architecture", IEEE Inyernational Conference, pp.277281.

[8]. National Institute of Standards and Technology, The NIST Definition of Cloud Computing, Information Technology Laboratory, 2009.

[9]. Rao, S, Rao, N \&Kumari, K 2009, "Cloud Computing: An Overview", Journal OF Theoretical And Applied Information Technology, Vol 9, No. 1, pp. 71-76.

[10]. Satyanarayana, S 2012, "CLOUD COMPUTING: SAAS", Journal of Computer Science and Telecommunications, Vol. 4, No. 4, pp. 76-79.

\section{BIOGRAPHY}

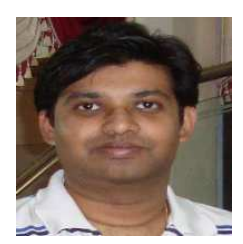

K.V.K Mahesh Kumar holds a B.E. (Bachelor of Engineering) in Computer Science from Osmania University, Graduate Diploma in Professional Computing \& Masters in ICT (Information and Communication Technology Management) from University of South Australia and is currently pursuing Ph.D. in Cloud Computing from Department of Computer Science and Engineering at Acharya Nagarjuna University. He has been involved in many diversified research projects and published several papers in international journals in the research areas of HCI (Human Computer Interaction), EHR (Electronic Health Record) systems, E-Business \& E-Commerce, Web 2.0 Social Networking, Project Management and Knowledge Management. 